\title{
Integrated Er/Si Schottky Photodetectors on the end facet of optical waveguides
}

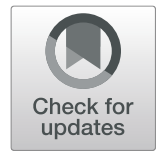

\author{
T. Crisci ${ }^{1}$, L. Moretti ${ }^{1}$, M. Gioffrè ${ }^{2}$, M. lodice ${ }^{2}$, G. Coppola ${ }^{2}$ and M. Casalino ${ }^{2^{*}}$ (D)
}

\begin{abstract}
In the last two decades there has been growing interest in silicon photonics and in the possibility to integrate new materials to overcome the silicon intrinsic limitations. Erbium has represented a viable solution for the realization of light sources at telecommunications wavelengths opening the path to the investigation of various photonic devices based on rare earth.

In this work we investigate a photodetector operating at $1550 \mathrm{~nm}$ whose detection mechanism is based on the internal photoemission effect through an Er/Si Schottky junction. The Er/Si junction has been carefully electrically characterized showing a potential barrier and cut-off wavelength of $0.59 \mathrm{eV}$ and $2105 \mathrm{~nm}$, respectively. Moreover, a responsivity of $0.62 \mathrm{~mA} / \mathrm{W}$ has been measured for a $3 \mu \mathrm{m}$-width waveguide at $1550 \mathrm{~nm}$ and at reverse voltage of -8 V. Finally, the noise equivalent power of the device has been evaluated as high as $0.53 \mathrm{nW} /(\mathrm{Hz})^{1 / 2}$ at $-8 \mathrm{~V}$. Even if device responsivity is still low, we believe that our insights may suggest Er/Si as a new platform for the integration of various optical functionalities on the same chip opening new frontiers in the field of low-cost silicon micro and nanophotonics.
\end{abstract}

Keywords: Silicon, Photodetector, Erbium, Internal photoemission effect, Schottky, Waveguide

\section{Introduction}

Photonics is the science of manipulation, detection and generation light by using optical components that include lasers, fiber-optics and electro-optical devices in several application fields. Integrated photonics is a branch of photonics in which the optical devices are integrated on a flat surface together the electronic devices, on the same platform. By using optical interconnections and devices intra-chip it's possible to overcome the bandwidth limitation and the high-power dissipation by electrical signals inside the semiconductor microchips, one of the most important problem in the modern computer system [1, 2]. A photonic integrated circuit (PIC) requires active and passive components, like couplers [3], interferometers [4], gratings [5], sources [6], beam splitters [7], detectors [8], sensors [9] and so on, which must be able to communicate with the electrical world,

\footnotetext{
* Correspondence: maurizio.casalino@na.imm.cnr.it

${ }^{2}$ Consiglio Nazionale delle Ricerche (CNR), Istituto per la Microelettronica e

Microsistemi (IMM), Via P. Castellino n. 111, 80131 Naples, Italy

Full list of author information is available at the end of the article
}

on and off-chip. To integrate different photonics components on an electronic platform it is now possible to use standard microelectronic foundry processes, with obvious advantages due to the re-use of the same electronic design tools and production technologies of the microelectronic sector. In this context Complementary Metal Oxide Semiconductor (CMOS) technological processes based on the use of substrates has become a key technology for the manufacture of optoelectronic devices [6, 10-14]. The high quality of SOI wafers, associated with the relatively low manufacturing costs (per unit area) of CMOS technology, makes them the ideal platform for the PIC fabrication [15]. These positive aspects are accompanied by some important physical problems. Silicon $(\mathrm{Si})$ is an indirect band semiconductor with a very low optical absorption at the NIR wavelengths, which makes it unsuitable for the realization of laser sources or NIR photodetectors.

An approach to overcome these problems is to use the integration of Si with Germanium (Ge). However, there are further technical problems because Ge is grown on 
Si substrates with epitaxial techniques $[16,17]$ which involve difficulties both in thermal budget and flatness [8], making complicated the monolithic integration of a Gebased PIC with electronic components on the same substrate. There are some examples in which it was possible to integrate photonics components, based on $\mathrm{Ge}$, on an electronic chip, but these components were fabricated separately and then subsequently mounted on a chip $[18,19]$. A further example consists in the manufacture of photodetectors (PDs) based on SiGe using a "zerochange" CMOS manufacturing process, with a percentage of Ge in the active part estimated between $25 \%$ and $35 \%$ [20], but in this case the operative wavelength is limited to maximum $1.2 \mu \mathrm{m}$ [21]. With this technique it was possible to realize a microprocessor with about 850 integrated photonic devices operating at $1180 \mathrm{~nm}$ with over 70 million transistors on the same chip [1], but at the moment no Ge-based PD's operating at $1550 \mathrm{~nm}$ have been realized. An alternative approach is the use of alternative materials exploiting their physical characteristics associated with innovative device geometries. An example is an IR PD's made by using lead sulphide quantum dots (PbS QDs) combined with a conjugated polymer (MEH-PPV). These devices showed a detection capability at wavelengths up to $1.355 \mu \mathrm{m}$ by varying the size of the QDs [22]. The PbS QDs were also deposited on a graphene film by spin-casting, in order to increase the sensitivity of a camera operating both in the visible and SWIR range [23]. However, these applications showed an inefficient integration with Si technology. At the moment an all-Si approach could be preferable [24].

An effect that can be used to increase the efficiency of Si-based PDs is the internal photoemission effect (IPE) that exploits the photo-excitation of carriers at an energy higher than the Schottky barrier of a metal/Si junction with consequent injection of carriers from metal to semiconductor. In recent years, numerous examples of PD using IPE in a metal/Si Schottky junction have been reported [25-28], showing however a responsivity of a few tens of $\mathrm{mA} / \mathrm{W}$. In particular the use of resonant structure has resulted beneficial in term of efficiency $[29,30]$. In more recent works a responsivity of $0.37 \mathrm{~A} / \mathrm{W}$ at $1550 \mathrm{~nm}$ was obtained using a graphene/silicon junction [31].

On the other hand, concerning light emission in $\mathrm{Si}$, recently considerable efforts have been made to realize erbium-doped silicon light sources that emit in the telecommunications band at $1550 \mathrm{~nm}$ in MOS geometry where the voltage between metal and silicon is used to excite the erbium (Er) crystals in the oxide gate [32]. These devices achieve efficiency of $10 \%$ but with a poor reliability. In the second version of these devices the silicon and erbium nanocrystals are dispersed together in the same oxide gate [32] showing a reduced efficiency but increased reliability. However, the energy back- transfer process that takes place between silicon and erbium reduces the emission at room temperature. Furthermore, an emissive decrease is noted as the current density increases, probably this behaviour is due to the absorption of free carriers and Auger processes. Many efforts are therefore necessary to improve the performance of these kinds of devices. Few studies have been done instead on the use of hybrid $\mathrm{Er} / \mathrm{Si}$ structures as near-infrared (NIR) PDs. There are some studies showing the possibility of making Schottky junctions with Er deposed on $\mathrm{Si}$ [33] and this capability has been recently employed to realize free-space illuminated $\mathrm{Er} / \mathrm{Si}$ Schottky PD operating at $1550 \mathrm{~nm}$ [34]. Therefore, it would be possible to easily integrate Er-based source and photodetector on the same chip, with considerable savings in terms of technology and costs. In fact, Erbium can be deposited with PVD techniques, such as sputtering, at low temperatures without compromising the functionality of pre-existing electronic/optic devices. Despite of these potentialities, no Er/Si Schottky PDs integrated in silicon waveguides have been reported, as far.

In this paper, we present an integrated PD whose detection mechanism is based on IPE through an $\mathrm{Er} / \mathrm{p}-\mathrm{Si}$ junction realized on the end facet of a SOI waveguide operating at $1550 \mathrm{~nm}$. First the IPE theory has been elucidated and the main models proposed in literature discussed. Then the Er/p-Si Schottky junction has been carefully electrically characterized in order to investigate its rectifying behaviour and to extract the electrical parameters in term of Schottky barrier, ideality factor and device series resistance. Finally, electro-optical measurements have been performed in order to achieve the responsivity at $1550 \mathrm{~nm}$ in function of the reverse bias applied, the spectral responsivity at $-8 \mathrm{~V}$ in the range $1540-1570 \mathrm{~nm}$ and the noise equivalent power (NEP).

\section{Methods}

\section{Internal photoemission effect theory}

IPE can be used in a Schottky junction for detecting a range of wavelengths corresponding to energies higher than the potential barrier at the metal/silicon interface. Metal is typically the active medium able to absorb the incoming radiation and to emit electrons (holes) through the Schottky junction into the $\mathrm{Si}$ conduction (valence) band. Energy band diagram for a metal/p-Si junction is sketched Fig. 1.

In order to quantify IPE-based PDs performance, a figure of merit named responsivity is typically used, it is the ratio between the photogenerated current $\mathrm{I}_{\mathrm{ph}}$ and the optical power $P_{\text {inc }}$ that affects the active absorbing medium:

$$
R=\frac{I_{p h}}{P_{\text {inc }}}=\frac{\lambda[n m]}{1242} \cdot \eta_{e} \quad[A / W]
$$




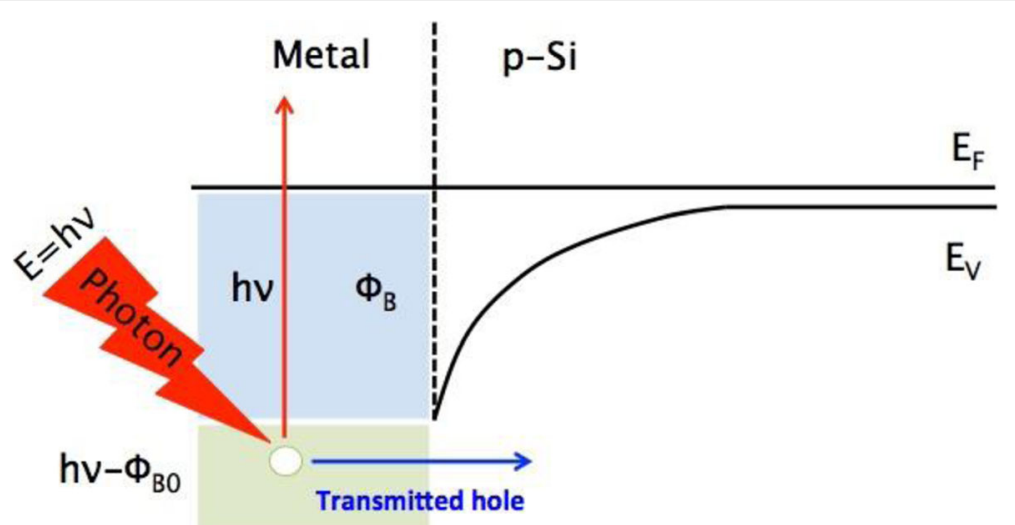

Fig. 1 Band diagram for a metal/p-type Si junction. $E_{F}$ is the Fermi level, $E_{V}$ is the Si valence band energy, $\Phi_{B}$ is the Schottky barrier at zero bias and $h v$ is the photon energy incoming on the metal

where $\lambda$ is the wavelength of the near-infrared (NIR) light in $\mathrm{nm}$ and $\eta_{\mathrm{e}}$ is the external quantum efficiency (number of carriers generated per incident photon). In addition, can be defined the internal quantum efficiency $\eta_{i}$, i.e., the number of charge carriers generated per absorbed photon. The optical absorbance of the active medium $\mathrm{S}$ allows for linking the external efficiency to the internal efficiency $\left(\eta_{e}=S \cdot \eta_{i}\right)$.

The first theoretical model was proposed by Fowler and focused on the emission of electrons from metal into vacuum [35]. The Fowler's theory was extended by Cohen, Vilms and Archer in 1960 [36] to the carrier emission from a metal into a semiconductor, extracting under the zero bias approximation the following formula for the internal quantum efficiency:

$$
\eta_{i}=C \cdot \frac{\left(h v-\phi_{B}\right)^{2}}{h v}
$$

where $h v$ is the energy photon, $\Phi_{B}$ is the Schottky barrier and $\mathrm{C}$ is the quantum efficiency coefficient. Subsequently Elabd and Kosonocky extended the Cohen, Vilms and Archer's theory [37] to the case of thin metal films where a multiplicative factor was introduced in order to take into account the increased emission probability due to multiple carrier scattering in the active layer [37]. Finally, Scales et al. [38] modified Elabd and Kosonocky's theory in order to calculate the emission probability through a double Schottky barrier. All the above-mentioned models were developed under the zero-temperature approximation. In the 1971 V.E. Vickers proposed a more complex and rigorous model able to describe IPE at any temperature [39]. More recently $M$. Casalino [40] showed that a slight modification of Eq. 2 leads to the following formula:

$$
\eta_{i}=\frac{1}{8 E_{F} h v}\left(\left(h v-\phi_{B}\right)^{2}+\frac{\left(k_{B} T \pi\right)^{2}}{3}\right)
$$

where $E_{F}$ is the metal Fermi level, $k_{B}$ is the Boltzmann and $\mathrm{T}$ is the absolute temperature. Eq. (3) is able to approximate the rigorous equation proposed by Vicker with an error within $10 \%$ when $\mathrm{h} v-\phi_{\mathrm{B} 0} \geq 0.035 \mathrm{eV}$, within $5 \%$ when $\mathrm{h} v-\phi_{\mathrm{B} 0} \geq 0.046 \mathrm{eV}$ and within $1 \%$ when $\mathrm{h} v$ $\phi_{\mathrm{B} 0} \geq 0.074 \mathrm{eV}$. The advantage of Eq. (3) is that it can be used at room temperature in the limit of the aforementioned errors.

\section{Device concept and fabrication}

The proposed device is a NIR PD based on IPE through an Er/p-Si Schottky integrated on the end facet of a SOI rib waveguide projected for single-mode operations, schematically illustrated in Fig. 2.

All the fabrication operations are compatible with $\mathrm{Si}$ technology. As first step, a standard RCA cleaning process on the SOI wafer was carried out. The waveguide was obtained via a combination of photolithographic processes and inductively coupled plasma reactive ion etching (ICP-RIE): first a trench of $220 \mathrm{~nm}$

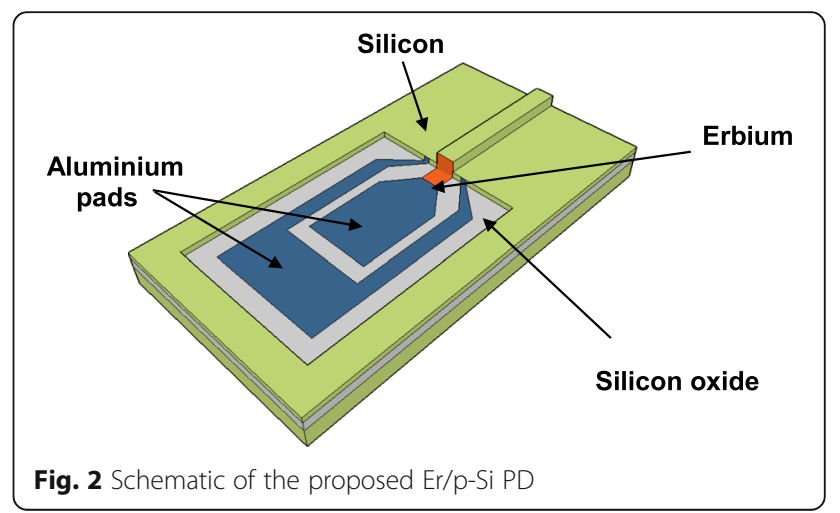



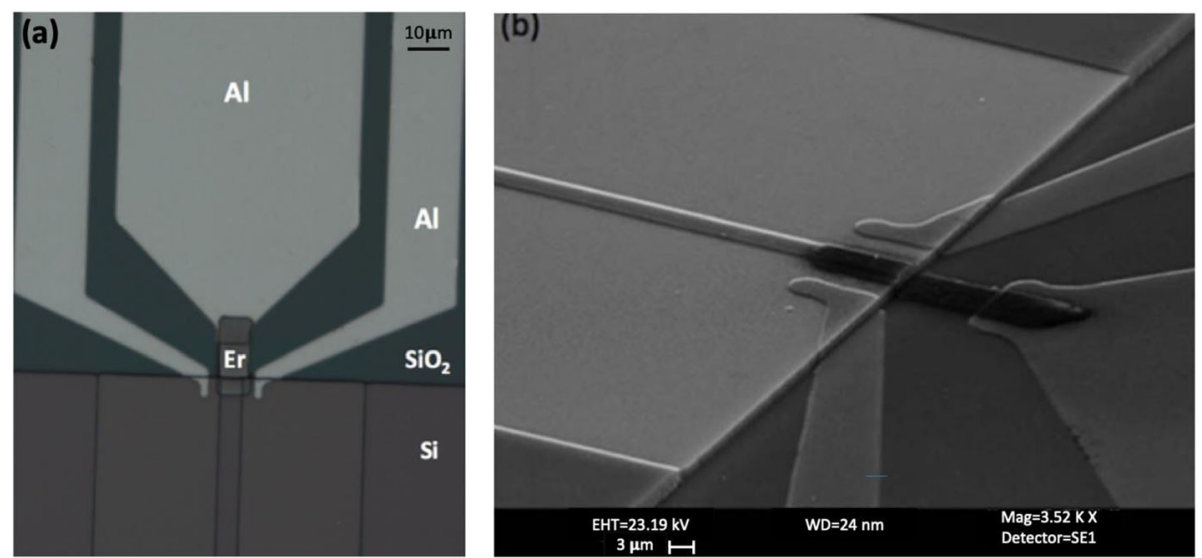

Fig. 3 (a) Top view image of the device taken by an optical microscope (5 $\mu$ m-width waveguide). (b) SEM image of the device

in the silicon top layer was realized in order to build the waveguide and then a second etching up to the buried oxide was performed to define the slab. The Schottky junction was formed through the deposition of thin layer of erbium on the vertical facet of the waveguide by sputtering. Erbium constitutes the active medium of the PD, absorbing the evanescent mode which propagates into the device. Finally, the Ohmic contacts, necessary for the collection of the photogenerated electrons in the active medium and emitted in the silicon, were realized by thermal evaporation of $150 \mathrm{~nm}$ thick aluminium pads, annealed for $30 \mathrm{~min}$ at $475^{\circ} \mathrm{C}$.

A top view image of the proposed photodetector, taken by an optical microscope and a SEM image of the Er/p-Si Schottky junction are shown in Fig. 3.

\section{Electrical characterization}

Current-voltage I-V measurements at room temperature were performed with the objective to show the rectifying behavior of the $\mathrm{Er} / \mathrm{Si}$ junction. The current was measured by varying the voltage across the junction from $-10 \mathrm{~V}$ to 1 V by taking advantage of a Source Meter (Agilent B2902A).

\section{Responsivity measurements and NEP evaluation}

Responsivity measurements were performed by using near-infrared light at telecom wavelengths around 1550 $\mathrm{nm}$ from a tunable laser (ANDO AQ4321D). The modulated laser beam was launched into the rib waveguide thanks to a tapered optical fiber. The photogenerated current was amplified with a transimpedance amplifier, which also provided a bias voltage to the junction, and measured by a lock-in technique. All photocurrent values are achieved as an average of 10 different measurements. The incident optical power was measured separately with a commercial calibrated InGaAs PD. In order to estimate the responsivity $\mathrm{R}$ of the devices it is important to evaluate the optical power incoming on the active absorbing layer Er. To this aim we evaluated the waveguide coupling and propagation optical losses by using the cut-back method [41]. The NEP [42], i.e. the optical signal power required to generate a photocurrent signal that is equal to the total noise current $i_{n}$ in the $\mathrm{PD}$ at a given wavelength and within a bandwidth of 1 $\mathrm{Hz}$, has been simply evaluated as NEP $=\mathrm{i}_{n} / \mathrm{R}$ [42].

\section{Results and discussion}

In this section both current-voltage and responsivity measurements on a $3 \mu \mathrm{m}$-width $\mathrm{Er} / \mathrm{p}$-Si waveguide photodetectors were reported and discussed.

Fig. 4 shows a forward current of $0.85 \mu \mathrm{A}$ at $1 \mathrm{~V}$, two order of magnitude higher than reverse current at $-1 \mathrm{~V}$, confirming the rectifying behavior of the $\mathrm{Er} / \mathrm{Si}$ junction. However, the forward curve seems characterized by a high series resistance, probably due to the reduced Er thickness that can be

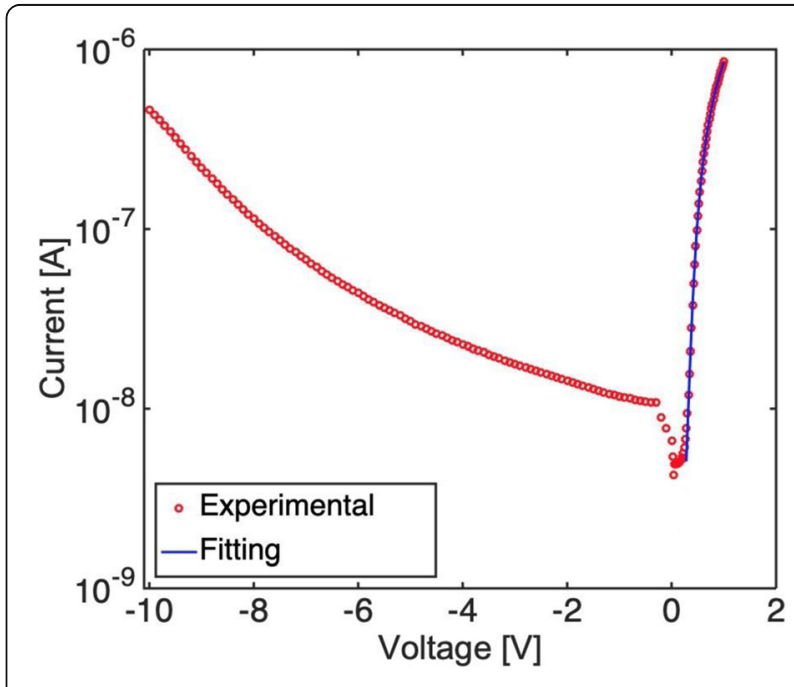

Fig. 4 I-V characteristic of the Er/p-Si junction at room temperature 
deposited on the vertical side of the rib waveguide by a nonconformal deposition technique as is the sputtering process.

By taking advantage of the canonical equation of the Schottky junction [43]:

$$
I=I_{d} \cdot\left(e^{\frac{V-R_{S} I}{\eta V_{t}}-1}\right)
$$

being the saturation current $I_{d}$ (named also dark current) written as:

$$
I_{d}=A_{P h} \cdot A^{*} \cdot T^{2} \cdot e^{-\frac{q \phi_{B}}{V_{t}}}
$$

where $A_{P h}$ is the area of the $\mathrm{Er} / \mathrm{Si}$ junction, $\mathrm{A}^{\prime \prime}$ is the Richardson constant $\left(32 \mathrm{~A} / \mathrm{cm}^{2} \mathrm{~K}^{2}\right), \mathrm{V}_{\mathrm{t}}=\mathrm{k}_{\mathrm{B}} \mathrm{T} / \mathrm{q}$ is the thermal voltage and $\mathrm{q}$ is the electron charge. It is possible to extract the Schottky barrier $\Phi_{B}$, the series resistance $R_{s}$ and the ideality coefficient $\eta$, by using a fitting procedure [44]. In this way a Schottky barrier $\Phi_{\mathrm{B}}$ of $(0.59 \pm 0.01) \mathrm{eV}$, corresponding to a cut-off wavelength of $(2105 \pm 36) \mathrm{nm}\left(\lambda_{\text {cut-off }}=1242 / \Phi_{\mathrm{B}}\right)$, was extrapolated while the series resistance and the ideality factor $\eta$ result as high as $(543 \pm 11) \mathrm{k} \Omega$ and $(1.94 \pm 0.05)$, respectively. It should be worth noting that the fitting procedure is applied only on the forward region of the IV characteristic where the Schottky barrier $\Phi_{\mathrm{B}}$ can be considered independent of the bias voltage and the diode current can be well-described by Eqs. (4) and (5). On the contrary in the reverse region of the IV characteristic, the Schottky

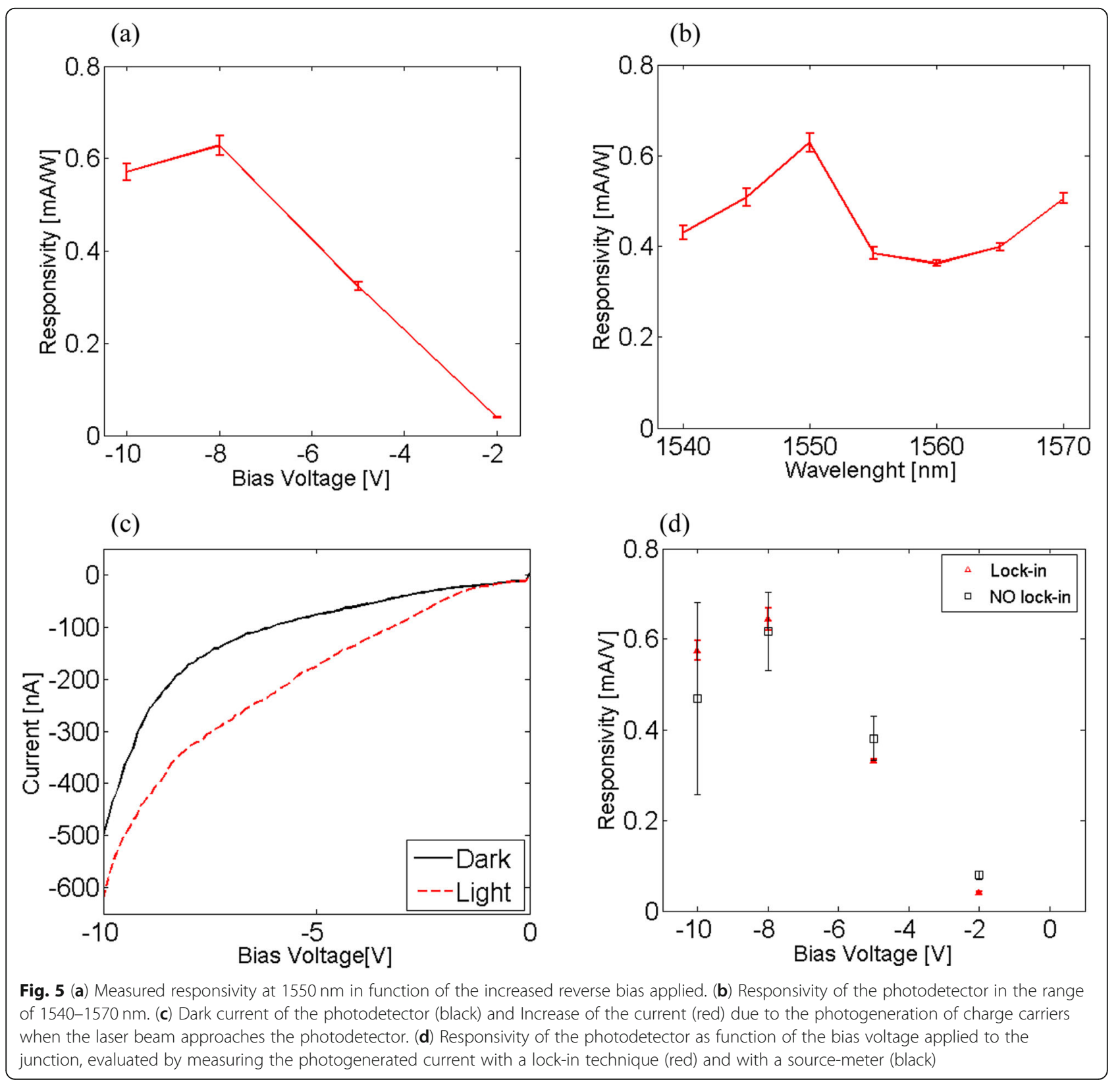


barrier $\Phi_{\mathrm{B}}$ decreases by increasing the negative bias voltage due to the very well-known image force effect [43], therefore this effect should be taken into account in Eq. (5) for an accurate prediction of the diode reverse current. Indeed, moving our attention on the reverse region of Fig. 4 it is possible to observe that the higher the reverse voltage applied, the higher the dark current $I_{d}$. The maximum dark current is $458 \mathrm{nA}$ at $-10 \mathrm{~V}$ of reverse bias applied.

Fig. 5(a) shows the measured responsivity in function of the increased reverse bias applied. Responsivity increases by increasing the reverse voltage applied up to $-8 \mathrm{~V}$, over $-8 \mathrm{~V}$ we can speculate that a saturation level seems reached, indeed the error bars at -8 an $-10 \mathrm{~V}$ are partially overlapped. The maximum measured responsivity is $0.62 \mathrm{~mA} / \mathrm{W}$. In addition, in Fig. 5(b) is reported the spectral responsivity in the range of 1540 $1570 \mathrm{~nm}$ (step of $0.05 \mathrm{~nm}$ ) at $-8 \mathrm{~V}$. Given that the waveguide was designed for operating at $1550 \mathrm{~nm}$ at this value corresponds to the maximum value of responsivity. Of course, the lock-in measurement technique allows for strongly reducing the noise current providing the measure of a very precise photocurrent signal.

In order to prove that the responsivity can be also measured without a lock-in technique, we repeated the same measurements by using a simple source meter and we performed measurements both in dark and under a continuous optical power of $256 \mu \mathrm{W}$ at $1550 \mathrm{~nm}$. All photocurrent values shown in Fig. 5(c) were achieved as an average of 5 different measurements. Fig. 5(c) clearly shows that the photocurrent signal is out of the noise margins and in Fig. 5(d) a comparison in term of measurement standard error provided by two techniques is reported.

Concerning the NEP evaluation, according to Llopis at al [45]. we suppose in a PD both Johnson and shot noise are higher than the $1 / \mathrm{f}$ noise at low frequencies. The noise current $i_{n}$ is, indeed, the sum of the shot and Johnson noise current $i_{n}=i_{s}+i_{j}$ where $i_{s}=\left(2 q\left(I_{p h}+\right.\right.$ $\left.\left.I_{\text {dark }}\right)\right)^{1 / 2}$ and $i_{j}=\left(\left(4 k_{B} T\right) / R_{e q}\right)^{1 / 2}$ with $R_{e q}$ the equivalent resistance of the photodetector when a reverse bias is applied. In our photodetector the Johnson noise current results to be over than 5 order of magnitude lower than the shot noise current, consequently, we compute the NEP as the ratio of the shot noise $i_{s}$ and the responsivity R. In Fig. 6 the NEP and the total noise current as function of the bias applied to the Schottky junction, are reported. It could be worth mentioning that when responsivity measurements are performed by the simple source-meter technique, Fig. 5(d) clearly shows that the higher the reverse voltage, the larger the error bars. This behavior is linked to the increase in dark current due to the lowering of the Schottky barrier $\Phi_{\mathrm{B}}$ (see Eq. 5) at reverse voltage generated by the well-known image force

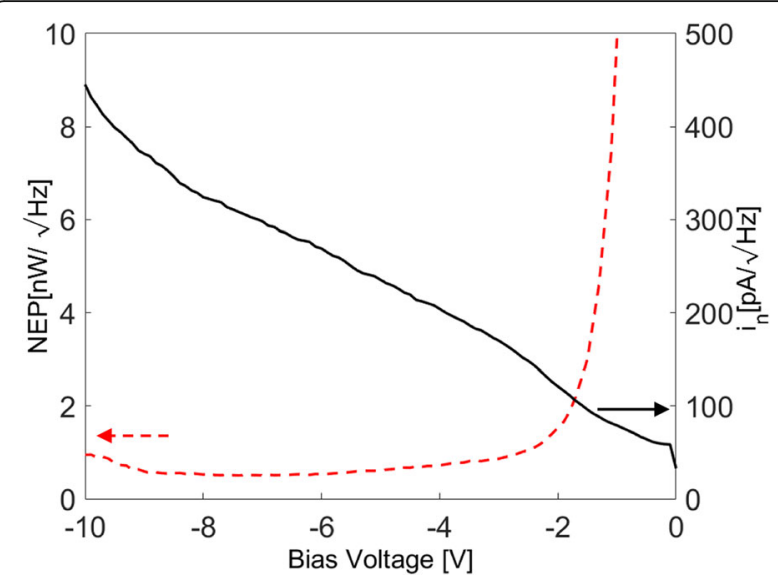

Fig. 6 NEP (red) and dark current (black) as function of the reverse bias applied to the Er/p-Si Schottky junction

effect [43]. Increased dark current leads to increased shot noise $i_{s}$ and, consequently, increased noise $i_{n}$ which represents the rms signal of the total noise fluctuations overlapped to the useful signal.

\section{Conclusions}

In this paper the fabrication and characterization of an Er/Si Schottky PDs integrated on the end facet of optical SOI waveguides and operating at $1550 \mathrm{~nm}$ has been reported. Electrical characterization shows that the junction obtained by replacing metal with Er can be well described by the canonical theory of the Schottky diodes with an ideality coefficient and potential barrier of 1.94 and $0.59 \mathrm{eV}$, respectively. The I-V curve shows the classical rectifying behavior with the forward current two orders of magnitude higher than the reverse one.

Concerning electro-optical characterization, we have used modulated NIR signal and a lock-in technique in order to achieve precise measurements of both the responsivity in function of the reverse bias applied at $1550 \mathrm{~nm}$ and the spectral responsivity in the range of $1540-1570 \mathrm{~nm}$ at $-8 \mathrm{~V}$. Moreover, we have shown that the same measurements could be obtained also without a lock-in technique even if with increased standard errors. A maximum responsivity of $0.62 \mathrm{~mA} / \mathrm{W}$ has been measured for a $3 \mu \mathrm{m}$-width waveguide at $1550 \mathrm{~nm}$ and at a reverse voltage of $-8 \mathrm{~V}$. Finally, the noise current and NEP have been evaluated and discussed.

In order to achieve an increase in responsivity two aspects could be considered: the reduction of the $\mathrm{Er} / \mathrm{Si}$ Schottky barrier and the enhancement of the optical absorption of the Er layer. Concerning the first option, it is worth noting that the $\mathrm{Er} / \mathrm{Si}$ potential barrier of $0.59 \mathrm{eV}$ extracted in this work is further high and limits the device efficiency as it is possible to understand by looking at Eqs. (2) or (3). However, as widely reported in 
literature, a rapid thermal annealing at limited temperature can contribute to reduce the $\mathrm{Er} / \mathrm{Si}$ Schottky barrier thanks to the formation of an erbium-silicide layer at $\mathrm{Si}$ interface [33]. Of course, in this case, the impact of the reduced Schottky barrier on the dark current should be evaluated. The second option concerns the possibility to enhance the optical absorption of the active medium by using well-designed integrated optical microcavities as disk or ring resonators, one simple possibility could be to realize Si Fabry-Peròt integrated microcavities whose mirrors are realized by etching deep trenches on the $\mathrm{Si} \mathrm{rib}$ waveguide as proposed by Barrios et al. [46].

Despite of low responsivity, we believe that this work can represent a proof-of-concept of PDs to be integrated with different Er-based optical functionalities in hybrid $\mathrm{Er} / \mathrm{Si}$ platforms opening new frontiers in the field of low-cost silicon photonics.

\section{Abbreviations}

CMOS: Complementary metal oxide Semiconductor; Er: Erbium;

Ge: Germanium; ICP-RIE: Inductively coupled plasma reactive ion etching; IPE: Internal photoemission effect; NEP: Noise equivalent power; NIR: Nearinfrared; PD: Photodetectors; PIC: Photonic integrated circuit; Si: Silicon; SOI: Silicon-on-insulator

\section{Acknowledgements}

Authors sincerely thank all technical and administrative staff of the Institute of Applied Sciences and Intelligent Systems in Naples for their constant support to the research activity, in particular: Francesco De Icco, Valeria Milo, Monica Gigliotti, Vincenzo Palmieri, Vincenzo Mosca, Maurizio Indolfi, Vitaliano Tufano, Domenico Passaro and Mauro Feliù.

\section{Authors' contributions}

Investigation, T.C., M.G., G.C., L.M. and M.C.; Project administration, M.C.; Writing-original draft, M.C., T.C. and M.G.; Writing-review \& editing, M.G., G.C., L.M., and M.I. All authors read and approved the final manuscript.

\section{Funding}

This work was partially funded from the European Union for ATTRACT under Grant Agreement no. 777222 and the Horizon 2020 Framework Programme for Research and Innovation (2014-2020).

\section{Availability of data and materials}

Please contact authors for data requests.

\section{Competing interests}

The authors declare that they have no competing interest.

\section{Author details}

${ }^{1}$ Dipartimento di Matematica e Fisica (DMF), Università degli Studi della Campania "Luigi Vanvitelli, Viale Lincoln n. 5, 81100 Caserta, Italy. ${ }^{2}$ Consiglio Nazionale delle Ricerche (CNR), Istituto per la Microelettronica e Microsistemi (IMM), Via P. Castellino n. 111, 80131 Naples, Italy.

Received: 31 October 2019 Accepted: 18 February 2020

Published online: 10 March 2020

\section{References}

1. Sun, C., Wade, M.T., Lee, Y., Orcutt, J.S., Alloatti, L., Georgas, M.S., Waterman, A.S., Shainline, J.M., Avizienis, R.R., Lin, S., Moss, B.R., Kumar, R., Pavanello, F., Atabaki, A.H., Cook, H.M., Ou, A.J., Leu, J.C., Chen, Y.-H., Asanović, K., Ram, R.J., Popović, M.A., Stojanović, V.M.: Single-chip microprocessor that communicates directly using light. Nat. 528(7583), 534-538 (2015)

2. Shacham, A., Bergman, K., Carloni, L.P.: Photonic networks-on-chip for future generations of chip multiprocessors. IEEE Trans. Comput. 57(9), 1246-1260 (2008)
3. Fang, Q., Song, J.F., Tao, S.H., Yu, M.B., Lo, G.Q., Kwong, D.L.: Low loss ( 6 $45 \mathrm{~dB} / \mathrm{cm}$ ) sub-micron polycrystalline silicon waveguide integrated with efficient SiON waveguide coupler. Opt. Express. 16(9), 6425-6432 (2008)

4. Mortada, B., Khalil, D., Omran, H., Medhat, M., Saadany, B.: Fully Integrated Mach-Zhender MEMS Interferometer With Two Complementary Outputs. Artic IEEE J Quantum Electron. 48(2), 244-251 (2012)

5. Kintaka, K., Kita, Y., Shimizu, K., Matsuoka, H., Ura, S., Nishii, J.: Cavityresonator-integrated grating input/output coupler for high-efficiency vertical coupling with a small aperture. Opt. Lett. 35(12), 1989-1991 (2010)

6. Liu, A., Hak, D., Rong, H., Paniccia, M., Cohen, O., Jones, R.: Optical Amplification and Lasing by Stimulated Raman Scattering in Silicon Waveguides. J Light Technol. 24(3), 1440 (2006)

7. Shen, B., Wang, P., Polson, R., Menon, R.: An integrated-nanophotonics polarization beamsplitter with $2.4 \times 2.4 \mu \mathrm{m}^{2}$ footprint. Nat. Photonics. 9(6), 378-382 (2015)

8. Wang, J., Lee, S.: Ge-photodetectors for Si-based optoelectronic integration. Sensors. 11(1), 696-718 (2011)

9. Terracciano, M., De Stefano, L., Borbone, N., Politi, J., Oliviero, G., Nici, F., Casalino, M., Piccialli, G., Dardano, P., Varra, M., Rea, I.: Solid phase synthesis of a thrombin binding aptamer on macroporous silica for label free optical quantification of thrombin. RSC Adv. 6(90), 86762-86769 (2016)

10. Rowe, L.K., Elsey, M., Tarr, N.G., Knights, A.P., Post, E.: CMOS-compatible optical rib waveguides defined by local oxidation of silicon. Electron. Lett. 43(7), 392-393 (2007)

11. Vivien, L., Pascal, D., Lardenois, S., Marris-Morini, D., Cassan, E., Grillot, F., Laval, S., Fédéli, J.-M., El Melhaoui, L.: Light Injection in SOI Microwaveguides Using High-Efficiency Grating Couplers. J. Light. Technol. 24(10), 3810-3815 (2006)

12. Xu, Q., Manipatruni, S., Schmidt, B., Shakya, J., Lipson, M.: 125 Gbit/s carrierinjection-based silicon micro-ring silicon modulators. Opt. Express. 15(2), 430 (2007)

13. Michael, C.P., Borselli, M., Johnson, T.J., Chrystal, C., Painter, O.: An optical fiber-taper probe for wafer-scale microphotonic device characterization. Opt. Express. 15(8), 4745 (2007)

14. Liu, A., Liao, L., Rubin, D., Nguyen, H., Ciftcioglu, B., Chetrit, Y., Izhaky, N., Paniccia, M.: High-speed optical modulation based on carrier depletion in a silicon waveguide. Opt. Express. 15(2), 660 (2007)

15. Tsybeskov, L., Lockwood, D.J., Ichikawa, M.: Silicon Photonics, "Silicon photonics: CMOS going optical". Proc. IEEE. 97(7), 1161-1165 (2009)

16. Eng, P.C., Song, S., Ping, B.: State-of-the-art photodetectors for optoelectronic integration at telecommunication wavelength. Nanophotonics. 4(1), 277-302 (2015)

17. Michel, J., Liu, J., Kimerling, L.C.: High-performance Ge-on-Si photodetectors. Nat. Photonics. 4(8), 527-534 (2010)

18. Alduino, A.: Demonstration of a high speed 4-channel integrated silicon photonics WDM link with hybrid silicon lasers. Paper presented at IEEE Hot Chips 22 Symposium (HCS 2010), Stanford (2010)

19. Narasimha, A., Analui, B., Balmater, E., Clark, A., Gal, T., Guckenberger, D., Gutierrez, S., Harrison, M., Ingram, R., Koumans, R., Kucharski, D., Leap, K., Liang, Y., Mekis, A., Mirsaidi, S., Peterson, M., Pham, T., Pinguet, T., Rines, D., Sadagopan, V., Sleboda, T.J., Song, D., Wang, Y., Welch, B., Witzens, J., Abdalla, S., Gloeckner, S., De Dobbelaere, P.: A 40-Gb/s QSFP optoelectronic transceiver in a $0.13 \mu \mathrm{m}$ CMOS silicon-on-insulator technology. In: OFC/ NFOEC 2008-2008 Conference on Optical Fiber Communication/National Fiber Optic Engineers Conference (2008)

20. Alloatti, L., Srinivasan, S.A., Orcutt, J.S., Ram, R.J.: Waveguide-coupled detector in zero-change complementary metal-oxide-semiconductor. Appl. Phys. Lett. 107(4), 041104 (2015)

21. Meng, H., Atabaki, A., Orcutt, J.S., Ram, R.J.: Sub-bandgap polysilicon photodetector in zero-change CMOS process for telecommunication wavelength. Opt. Express. 23(25), 32643-32653 (2015)

22. Mcdonald, S.A., Konstantatos, G., Zhang, S., Cyr, P.W., Klem, E.J.D., Sargent, L. Solution-Processed PbS Quantum Dot Infrared Photodetectors and Photovoltaics, Nature materials 4, 138-142 (2005).

23. Goossens, S., Navickaite, G., Monasterio, C., Gupta, S., Piqueras, J.J., Pérez, R., Burwell, G., Nikitskiy, I., Lasanta, T., Galán, T., Puma, E., Centeno, A., Pesquera, A., Zurutuza, A., Konstantatos, G., Koppens, F.: Broadband image sensor array based on graphene-CMOS integration. Nat. Photonics. 11(6), 366-371 (2017)

24. Casalino, M., Coppola, G., De La Rue, R.M., Logan, D.F.: State-of-the-art allsilicon sub-bandgap photodetectors at telecom and datacom wavelengths. Laser Photonics Rev. 10(6), 895-921 (2016) 
25. Zhu, S., Yu, M.B., Lo, G.Q., Kwong, D.L.: Near-infrared waveguide-based nickel silicide Schottky-barrier photodetector for optical communications. Appl. Phys. Lett. 92(8), 081103 (2008)

26. Casalino, M., lodice, M., Sirleto, L., Rao, S., Rendina, I., Coppola, G.: Low dark current silicon-on-insulator waveguide metal-semiconductor-metalphotodetector based on internal photoemissions at $1550 \mathrm{~nm}$. J. Appl. Phys. 114(15), 153103 (2013)

27. Berini, P.: Surface plasmon photodetectors and their applications. Laser Photonics Rev. 8(2), 197-220 (2014)

28. Casalino, M., Sirleto, L., Moretti, L., Gioffr, M., Coppola, G., Rendina, I.: Silicon resonant cavity enhanced photodetector based on the internal photoemission effect at $1.55 \mu \mathrm{m}$ : Fabrication and characterization. Appl. Phys. Lett. 92(25), 251104 (2008)

29. Casalino, M., Russo, R., Russo, C., Ciajolo, A., Di Gennaro, E., lodice, M., Coppola, G.: Free-Space Graphene/Silicon Photodetectors Operating at 2 Micron 5, 4577-4585 (2018)

30. Casalino, M.: Design of Resonant Cavity-Enhanced Schottky Graphene/ Silicon Photodetectors at $1550 \mathrm{~nm}$. J. Light. Technol. 36(9), 1766-1774 (2018)

31. Goykhman, I., Sassi, U., Desiatov, B., Mazurski, N., Milana, S., De Fazio, D., Eiden, A., Khurgin, J., Shappir, J., Levy, U., Ferrari, A.C.: On-Chip integrated, silicon-Graphene Plasmonic Schottky Photodetector with high Responsivity and avalanche Photogain. Nano Lett. 16(5), 3005-3013 (2016)

32. Castagna, M.E., Coffa, S., Caristia, L., Messina, A., Bongiorno, C.: Quantum dot materials and devices for light emission in silicon. Paper presented at 32nd European Solid-State Device Research Conference, Firenze (2002)

33. M. Jang, Y. Kim, J. Shin, and S. Lee, "Formation of erbium-silicide as source and drain for decananometer-scale Schottky barrier metal-oxidesemiconductor field-effect transistors," in Materials Science and Engineering B: Solid-State Materials for Advanced Technology, 114-115(SPEC. ISS)., 51-55 (2004)

34. Gioffré, M., Coppola, G., lodice, M., Casalino, M.: Integrable Near-Infrared Photodetectors Based on Hybrid Erbium/Silicon Junctions. Sensors 18(11), 3755 (2018)

35. Fowler, R.H.: The analysis of photoelectric sensitivity curves for clean metals at various temperatures. Phys. Rev. 38(1), 45-56 (1931)

36. Co, J., Vilms, S.J., Archer, R.J.: Investigation of semiconductor schottky barriers for optical detection and cathodic emission 00 final report / nfeb25m (1967)

37. Elabd, W.F.K.H.: Theory and Measurements of Photoresponse for Thin PdzSi and PtSi Infrared Schottky-Barrier Detectors with Optical Cavity (1982)

38. Scales, C., Berini, P.: Thin-film schottky barrier photodetector models. IEEE J. Quantum Electron. 46(5), 633-643 (2010)

39. Vickers, V.E.: Model of Schottky Barrier Hot-Electron-Mode Photodetection. Appl. Optics. 10(9), 2190 (1971)

40. Casalino, M.: Internal photoemission theory: Comments and theoretical limitations on the performance of near-infrared silicon Schottky photodetectors. IEEE J. Quantum Electron. 52(4), 4000110 (2016)

41. Reed, G.T., Knights, A.P.: Silicon Photonics: an Introduction. Wiley, Chichester (2004)

42. Kasap, S.O.: Optoelectronics and Photonics: Principles and Practices. Prentice Hall Englewood Cliffs, New Jersey (2001)

43. Sze, S.M., Ng, K.K.: Physics of Semiconductor Devices. John Wiley \& Sons, Inc., New Jersey (2006)

44. Lien, C.D., So, F.C.T., Nicolet, M.A.: An improved forward I-V method for nonideal Schottky diodes with high series resistance. IEEE Trans. Electron Devices. 31(10), 1502-1503 (1984)

45. Llopis, O., Azaizia, S., Saleh, K., Slimane, A.A., Fernandez, A.: Photodiode 1/f Noise and Other Types of less Known Baseband Noises in Optical Telecommunications Devices. In: IEEE 22nd International Conference on Noise and Fluctuations, ICNF 2013 (2013)

46. Barrios, C.A., Rosa de Almeida, V., Lipson, M.: Low-power-consumption shortlength and high-modulation-depth silicon electrooptic modulator. IEEE J. Lightwave Technol. 21, 1089-1098 (2003)

\section{Publisher's Note}

Springer Nature remains neutral with regard to jurisdictional claims in published maps and institutional affiliations.

\section{Submit your manuscript to a SpringerOpen ${ }^{\circ}$ journal and benefit from:}

- Convenient online submission

- Rigorous peer review

- Open access: articles freely available online

- High visibility within the field

- Retaining the copyright to your article

Submit your next manuscript at $\boldsymbol{\nabla}$ springeropen.com 